\title{
An experimental comparison of conventional two-bank and novel four-bank dynamic MLC tracking
}

\author{
G A Davies, P Clowes, D McQuaid, P M Evans, S Webb and \\ G Poludniowski \\ Joint Department of Physics, Institute of Cancer Research and Royal Marsden NHS \\ Foundation Trust, Downs Road, Sutton, Surrey, SM2 5PT \\ E-mail: gemma.davies@icr.ac.uk
}

\begin{abstract}
The AccuLeaf mMLC featuring four multileaf-collimator (MLC) banks has been used for the first time for an experimental comparison of conventional twobank with novel four-bank dynamic MLC tracking of a two-dimensional sinusoidal respiratory motion. This comparison was performed for a square aperture, and for three conformal treatment apertures from clinical radiotherapy lung cancer patients. The system latency of this prototype tracking system was evaluated and found to be $1.0 \mathrm{~s}$ and the frequency at which MLC positions could be updated, $1 \mathrm{~Hz}$, and therefore accurate MLC tracking of irregular patient motion would be difficult with the system in its current form. The MLC leaf velocity required for two-bank-MLC and fourbank-MLC tracking was evaluated for the apertures studied and a substantial decrease was found in the maximum MLC velocity required when four-banks were used for tracking rather than two. A dosimetric comparison of the two techniques was also performed and minimal difference was found between two-bank-MLC and four-bankMLC tracking. The use of four MLC banks for dynamic MLC tracking is shown to be potentially advantageous for increasing the delivery efficiency compared with twobank-MLC tracking where difficulties are encountered if large leaf shifts are required to track motion perpendicular to the direction of leaf travel.
\end{abstract}

\section{Introduction}

Dynamic multileaf-collimator (MLC) tracking has been demonstrated to be a promising technique for compensation of intrafraction motion of the treatment target during radiotherapy in numerous research studies (Sawant et al. 2008, Keall et al. 2011, Krauss et al. 2012). Despite this, tracking respiratory motion with a conventional two-bank MLC design has been shown to result in reduced treatment efficiency and imperfect reconstruction of the intended dose distribution, which several authors have attributed to the difficultly in tracking a target motion vector perpendicular to the direction of leaf travel (McQuaid \& Webb 2006, Krauss et al. 2011b, Yoon et al. 2011). When tracking a motion perpendicular to the direction of MLC leaf travel, it is necessary to shift the position of each MLC leaf in the bank to its neighbour. If the distance 
between the position of adjacent leaves is large, and the maximum velocity of the MLC leaves is insufficient to perform this shift in the time interval desired, beam-holds may be required in order for the leaves to move to the relevant position, resulting in an increased treatment time. There are also dosimetric consequences due to the finite width of each MLC leaf which means it is impossible to perfectly recreate the planned treatment aperture if a shift perpendicular to the direction of leaf travel is required.

A possible solution to these difficulties could be the introduction of a second set of MLC banks whose direction of travel is perpendicular to the first set. The AccuLeaf micro multileaf-collimator (mMLC) developed by Azimuth Therapy Ltd features these extra banks, and is used here for the first time to compare conventional MLC tracking of conformal treatment apertures with two MLC banks with MLC tracking with four MLC banks. It has been demonstrated that the four-bank AccuLeaf mMLC offers increased conformality and reduced penumbra for aperture formation (Liu et al. 2008), and also that it can confer advantage when sequencing 2D intensity-modulated beams for stepand-shoot intensity-modulated radiotherapy (Webb 2012), but the performance of the AccuLeaf mMLC for dynamic MLC tracking has not previously been investigated.

The experiments presented in this study investigated the maximum leaf velocity required for two-bank-MLC and four-bank-MLC tracking of realistic conformal treatment apertures, and a dosimetric evaluation of the two techniques was performed to see if the use of additional MLC banks improves the delivery accuracy of the planned dose distribution.

\section{Method}

\subsection{Dynamic $M L C$ tracking control system}

The current clinical AccuLeaf control system does not support real-time dynamic MLC tracking therefore, to explore this motion-compensation technique with the AccuLeaf mMLC, comprehensive specifications were given to the manufacturers to provide certain functions within a $\mathrm{C}++$ dynamic-linked-library (DLL), which could be used to communicate with the AccuLeaf mMLC. This enabled control over each individual MLC leaf and adjustment of the planned MLC positions in the clinical AccuLeaf control system from an external source. Target motion simulation was performed using a motion platform built in-house featuring a Galil DMC-4060 (Galil Motion Control, Inc.) motion controller, for which a $\mathrm{C}++$ DLL was also available, therefore it was possible to develop a prototype tracking control system capable of adjusting the AccuLeaf mMLC positions dynamically, and communicating with the motion platform. With the use of a fibre-optic optical gating interface, connected between the motion platform and the linear accelerator, it was also possible to inhibit the linear accelerator pulse-repetition frequency (PRF) until the motion platform began motion, and thus gate the x-ray beam.

Preliminary tests with the DLL provided by the manufacturers indicated that, in order for successful aperture formation, it was not possible to send updates to the device 
at a frequency greater than $1 \mathrm{~Hz}$, therefore the MLC positions were adjusted to follow a breathing trajectory at $1 \mathrm{~s}$ intervals. Typical time periods of respiratory motion for lung cancer patients were found to be in the range of $4-6.6 \mathrm{~s}$ with an average of $3.6 \mathrm{~s}$ in a study of twenty patients presented by Seppenwolde (2002). Therefore, for dynamic MLC tracking of respiratory motion, the update frequency of this prototype tracking system is insufficient, and leads to coarse sampling of a typical breathing trajectory. To overcome this problem and investigate purely the dosimetric differences between twobank-MLC and four-bank-MLC tracking, a time period substantially longer than those observed clinically was chosen such that MLC tracking experiments with this prototype system were possible. This is described in more detail in section 2.3. The flowchart in figure 1 illustrates the process followed by the tracking control system.

\subsection{Conformal treatment apertures for tracking}

Each bank of the AccuLeaf mMLC contains 24 leaves: 14 central leaves of physical width $2.1 \mathrm{~mm}$ and 10 outer leaves of physical width $3.6 \mathrm{~mm}$. One pair of MLC banks is mounted above the other and therefore the leaves of each pair of banks have different projected widths at isocentre. The leaf width at isocentre is dependent on the particular mounting configuration, and for the Elekta Synergy linear accelerator used in this study, the set of banks closest to the patient (groups 1 and 2) have a projected leaf width at isocentre of $5.2 \mathrm{~mm}$ and $3.0 \mathrm{~mm}$ for the wide and narrow leaves respectively, and for the sets of banks farthest from the patient (groups 3 and 4), the corresponding values are $5.7 \mathrm{~mm}$ and $3.3 \mathrm{~mm}$.

Four-bank-MLC versus two-bank-MLC tracking was compared for four conformal treatment apertures: a $30 \mathrm{~mm}$ square aperture and three apertures taken from plans for lung cancer patients. In order for the AccuLeaf to mimic a two-bank MLC, the banks not being used for aperture formation were parked at the periphery of the mMLC device. A minimum $1 \mathrm{~mm}$ gap at isocentre was left between the banks being used for aperture formation to avoid collisions. The square apertures are illustrated in figure 2 .

The three apertures from clinical lung cancer patients were derived by extracting each MLC aperture contour from the original plans created using an Elekta Beam Modulator MLC, and then fitting the AccuLeaf mMLC leaves to this contour. As the AccuLeaf mMLC has a maximum field size at isocentre of approximately $100 \mathrm{~mm}$ $\times 100 \mathrm{~mm}$, patients 2 and 3 had to have a size-reducing scaling factor applied to the contour of 0.5 and 0.4 respectively so that it was possible to form the apertures with the AccuLeaf mMLC. The apertures for patient 3 are shown in figure 3. For all patients, the two-bank apertures were formed using the leaves from groups 3 and 4 , chosen to maximise the number of MLC leaves touching the contour. The leaves not required from these banks were parked at the mid-point of the last pair of open leaves at the superior and inferior end of the contour. For the two-bank square aperture, groups 1 and 2 were used for aperture formation, and the leaves of groups 3 and 4 were parked at the periphery of the mMLC device, as these leaf groups have the largest leaf width 


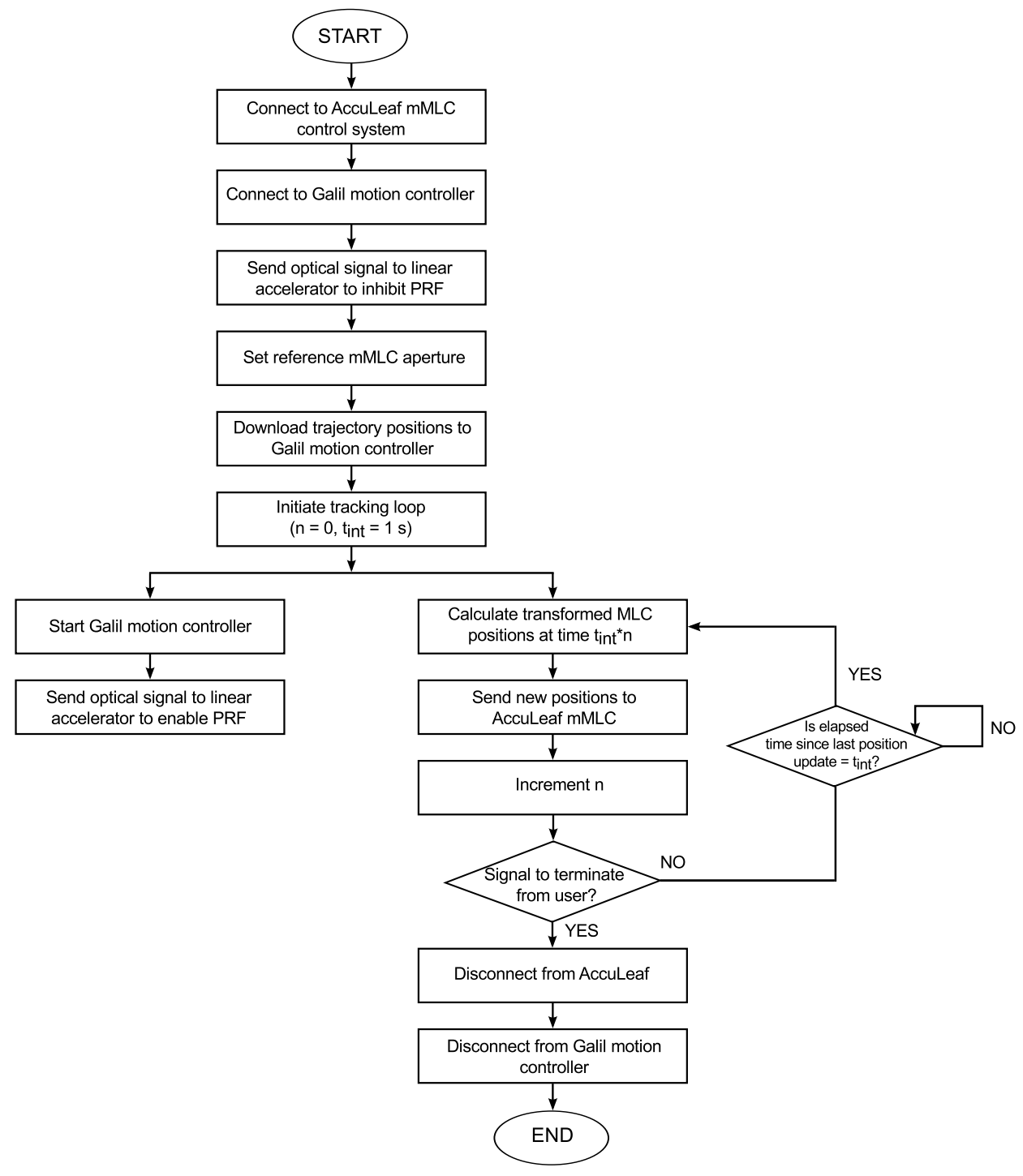

Figure 1. Flowchart to illustrate the program developed for tracking with the AccuLeaf mMLC. The interval time for sending new positions to the mMLC is represented by $t_{i n t}$, and $n$ is an integer, which is incremented each time a new position is sent to the mMLC.

at isocentre. The four-bank apertures were formed with the aim of minimising the distance between adjacent leaves. The gradient at the superior and inferior end of the contour was calculated and depending on the magnitude of the gradient then either the transverse leaves of group 3 and 4 were fitted to the contour or the longitudinal leaves of groups 1 and 2 .

For all apertures investigated, the treatment field was set to be a $6 \mathrm{MV}$ beam of 300 $\mathrm{MU}$ at gantry angle $0^{\circ}$ with a dose rate of $300 \mathrm{MU} \mathrm{min}^{-1}$. 


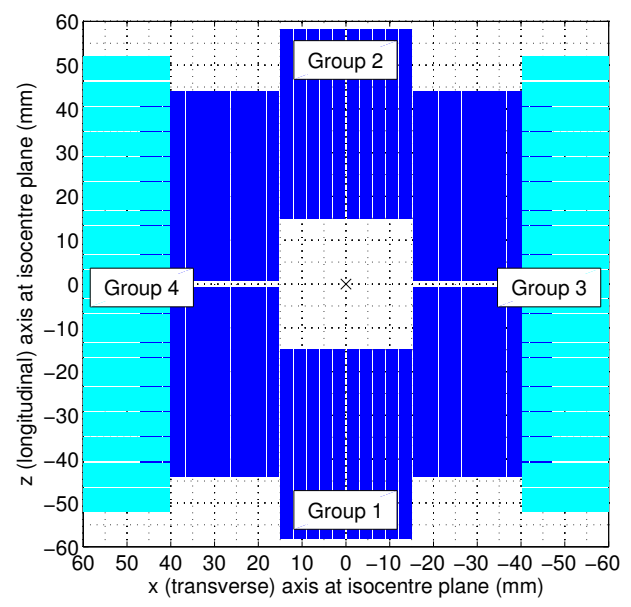

(a)

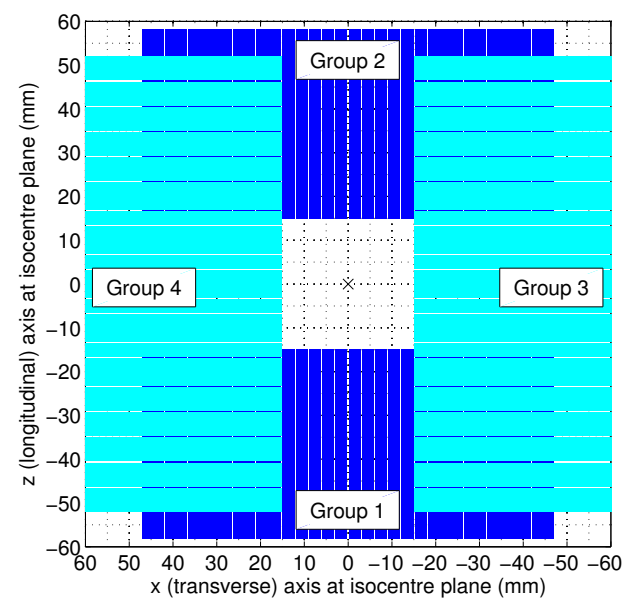

(b)

Figure 2. A $30 \mathrm{~mm}$ width square aperture formed with (a) two MLC banks and (b) four MLC banks.

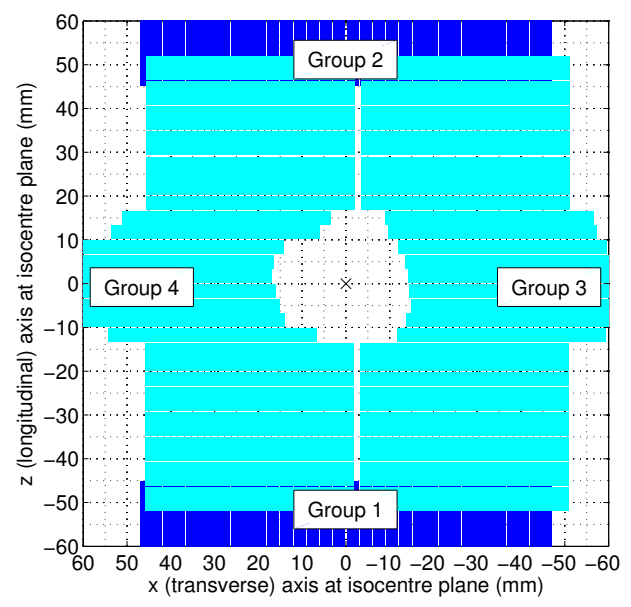

(a)

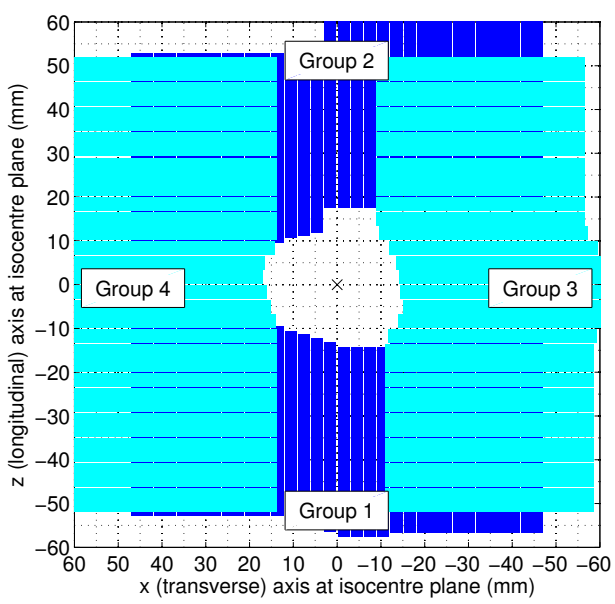

(b)

Figure 3. Conformal aperture for patient 3 formed with (a) two MLC banks and (b) four MLC banks.

\subsection{Simulation of the respiratory-motion trajectory}

For the MLC tracking experiments, a two-dimensional Lujan (2003) respiratory motion trajectory was modelled:

$$
\begin{aligned}
& x_{\text {target }}(t)=x_{0}-A_{x} \cos ^{6}\left(\frac{\pi t}{\tau}+\frac{\pi}{5}\right) \\
& z_{\text {target }}(t)=z_{0}+A_{z} \cos ^{6}\left(\frac{\pi t}{\tau}+\frac{3 \pi}{5}\right)
\end{aligned}
$$


where $x$ is parallel to the direction of motion of leaf groups 3 and 4 and $z$ is parallel to the direction of motion of leaf groups 1 and 2 (see figures 2 and 3). The amplitudes, $A_{x}=A_{z}=20 \mathrm{~mm}$, the time period of motion, $\tau=20 \mathrm{~s}$ and the rest position, $x_{0}=z_{0}=$ 0 . The time period of $20 \mathrm{~s}$ was chosen such that with the update frequency of $1 \mathrm{~Hz}$, the breathing trajectory was sampled twenty times. This would be analogous to a $4 \mathrm{~s}$ time period sampled twenty times with an update frequency of $5 \mathrm{~Hz}$, which would be a more desirable update frequency for a real-time MLC tracking system, but was not yet possible with this prototype.

The motion platform described in section 2.1 was programmed to move with the $2 \mathrm{D}$ motion described in (1) and (2). Figure 4 shows the experimental set-up and the axes of motion with respect to the equipment.

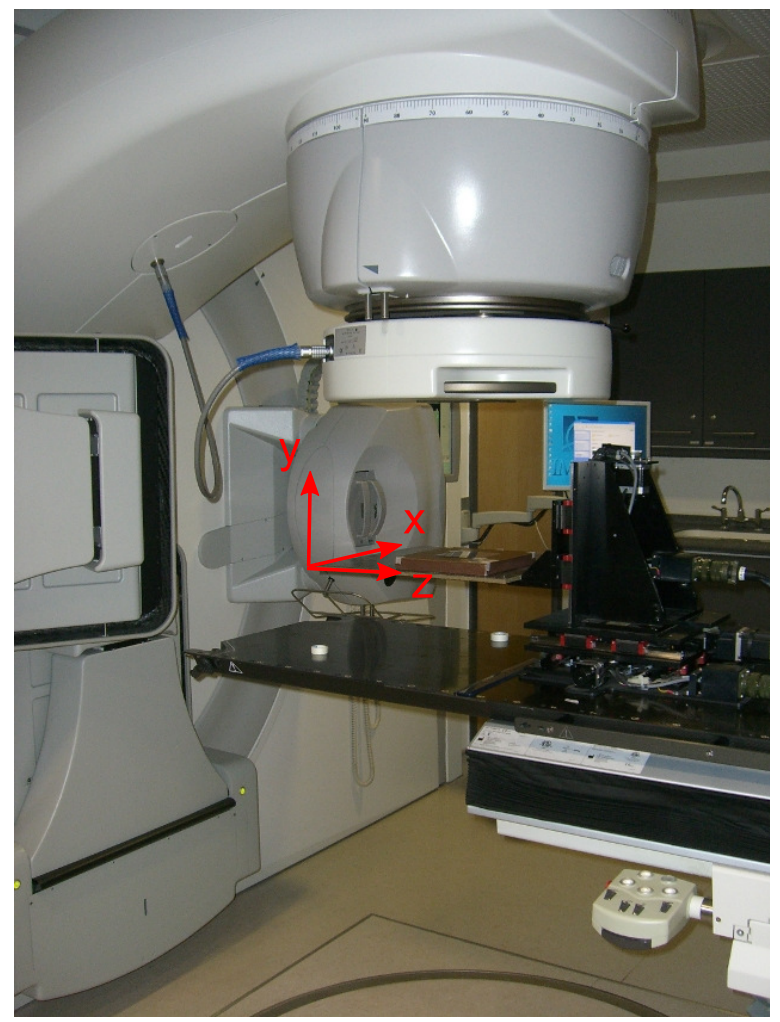

Figure 4. Photograph of the experimental set-up: the motion platform is shown mounted on the linear accelerator couch and the AccuLeaf mMLC is shown attached to the head of the Elekta linear accelerator via the accessory mount. The orientation of the axes of motion are labelled in red.

\subsection{Algorithm for adjusting MLC leaf positions}

To track motion parallel to the direction of leaf travel, the positions, $z_{k}$, of the $k$ MLC leaves in groups 1 and 2, were transformed using:

$$
z_{k}^{\prime}=z_{k}+z_{\text {target }}(t)
$$


and the positions, $x_{k}$, of the $k$ MLC leaves in groups 3 and 4 can be transformed using:

$$
x_{k}^{\prime}=x_{k}+x_{\text {target }}(t)
$$

where a prime indicates that the coordinate has been transformed to compensate for motion.

To compensate for motion perpendicular to the direction of leaf travel, a linear interpolation approach was taken, such that the contour of the planned aperture was shifted according to the magnitude and direction of the target motion, and then the centre of each MLC leaf edge was fitted to its intercept with this new, transformed aperture contour. This is illustrated in figure 5 for the transverse leaves of group 4 .
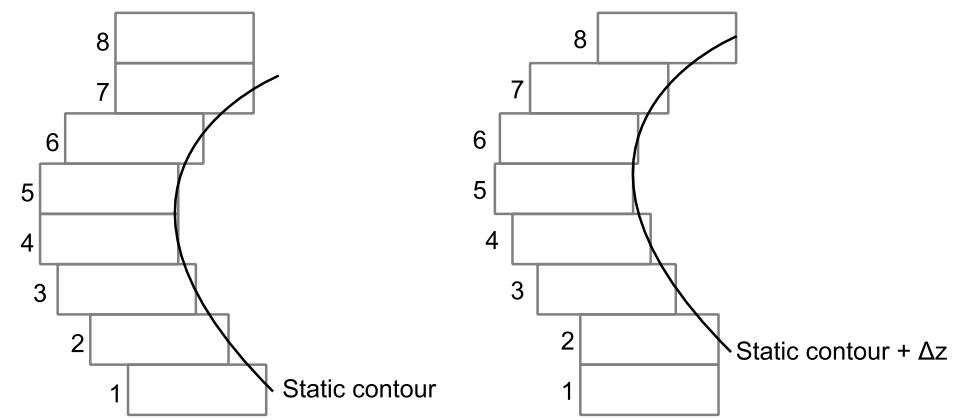

Figure 5. Schematic illustrating how the MLC leaves were adjusted to compensate for motion perpendicular to the direction of leaf travel. The planned aperture was defined by a static contour, and when there was motion e.g. in this case, $\Delta z$, the contour was shifted, and the leaves were refitted such that the centre of each MLC leaf edge was aligned with the shifted contour.

\subsection{Assessment of the latency of the tracking system}

The developed MLC tracking control system initiates a timer to start the tracking process on a dedicated thread, and then the motion controller is started immediately afterwards. The tracking system therefore has an inherent system latency, or response time, due to the sequential nature of this process, and also the time taken for calculation and transmission of the new leaf positions and leaf motion. To determine this latency, a multi-frame acquisition of a radio-opaque marker mounted on to the moving motion platform with the square aperture in MLC tracking mode was taken using the electronic portal imaging device (EPID), at a rate of 2.2 frames per second. These images were imported into MATLAB ${ }^{\circledR}$ (The MathWorks, Inc.), and using a cross-correlation technique, the co-ordinates of the centre of the radio-opaque marker, and the geometric centre of the aperture determined for each frame, resulting in a trajectory for the radioopaque marker and aperture over the multi-frame acquisition. A non-linear least-squares 
curve-fitting technique was used to fit the Lujan function to these trajectories and thus determine the phase difference.

To estimate the uncertainty in this process, the EPID acquisition was repeated twice, and the curve-fitting process was performed for six subset trajectories, generated by splitting up the trajectories from the two EPID acquisitions into six individual breathing cycles. The mean phase difference was taken to be the system latency value, and the standard deviation, the uncertainty in this process.

As the motion being studied here was sinusoidal, the phase difference between the movement of the motion platform and the mMLC leaves moving to respond to the motion was removed by delaying the movement of the motion platform by the system latency time. For unknown irregular motion, it would be necessary to employ predictahead algorithms such as those investigated by Krauss (2011a) and Ruan (2010) but these were not needed for the present study.

\subsection{Film measurements}

Gafchromic ${ }^{\circledR}$ EBT film mounted between two sheets of solid water on the motion

platform, placed at isocentre plane at a depth of $15 \mathrm{~mm}$ (figure 4), was used to evaluate the dosimetric performance of MLC tracking with the AccuLeaf mMLC. For both four-bank-MLC and two-bank-MLC tracking, a film was taken of the static conformal treatment aperture, with the static motion platform (reference film). Following this, a film was taken of the static conformal treatment aperture with the moving motion platform (no-tracking film) and finally, a film was taken of the tracking treatment aperture, with the moving motion platform (MLC-tracking film).

Both the no-tracking film and the MLC-tracking film were compared with the reference film, by calculation of root-mean-square percentage difference, and also with a gamma analysis (Low et al. 1998) using a tolerance level of $3 \%$ and $3 \mathrm{~mm}$.

\section{Results}

\subsection{Assessment of the latency of the system}

The $\mathrm{x}$-axis trajectories of the geometric centre of the aperture and the radio-opaque marker derived from the EPID multi-frame acquisition without latency compensation are shown in figure 6(a). A phase difference between the two Lujan trajectories was observed, with the trajectory of the radio-opaque marker leading the aperture trajectory. This phase difference was found to correspond to an absolute time difference of $1.0 \pm$ $0.1 \mathrm{~s}$. Compensation for the system latency was achieved by delaying the movement of the motion platform, and the results of the repeat EPID multi-frame acquisition are shown in figure $6(\mathrm{~b})$. It can been seen that the phase difference between the trajectory of the aperture and the radio-opaque marker is now close to zero. 


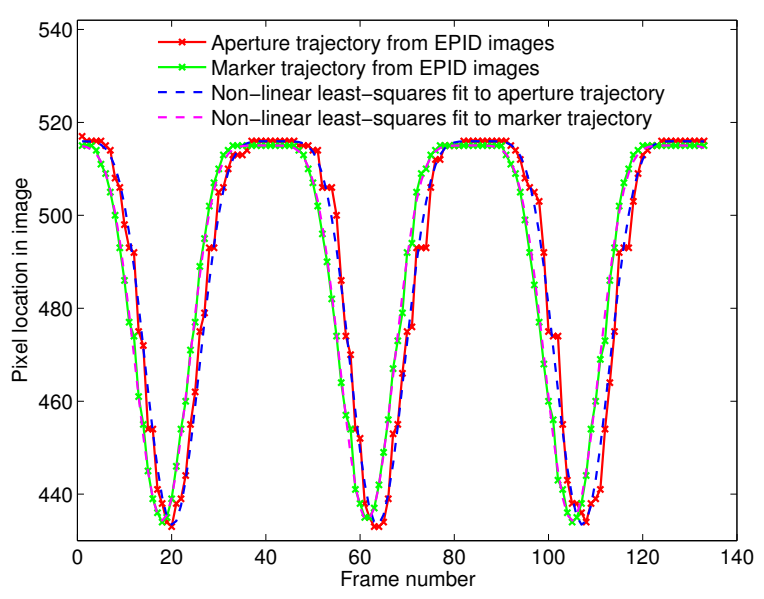

(a)

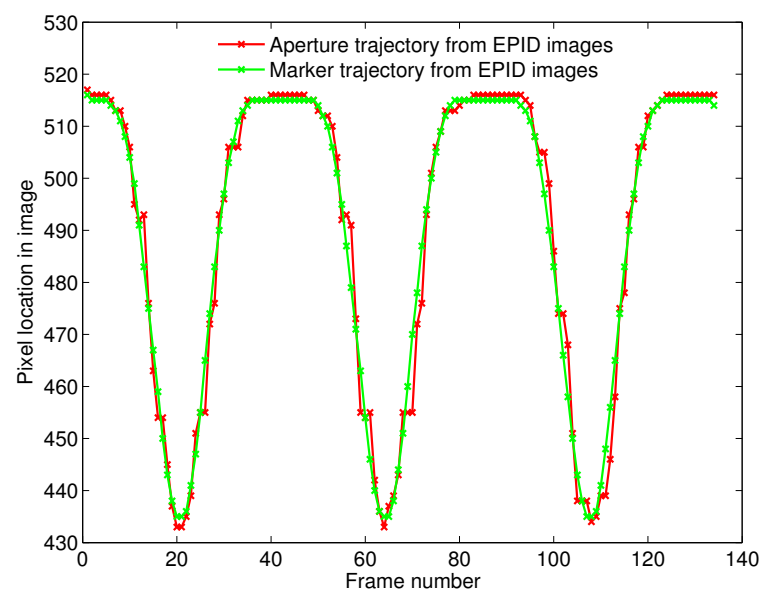

(b)

Figure 6. Trajectories of the geometric centre of the MLC aperture and the radioopaque marker generated from EPID images. For brevity, only the trajectories in the $\mathrm{x}$ (transverse) direction are shown. Figure 6(a) shows the trajectories and the corresponding Lujan fit without latency compensation. Figure 6(b) shows the analogous trajectories for a repeat acquisition with a delay to the movement of the motion platform to compensate for the system latency.

\subsection{Film measurements}

The film results of the four-bank aperture for patient 3 (see figure 3(b) for aperture) are given in figure 7. Figure 7(a) shows the film taken with the static MLC aperture and the static motion platform; this is the desired dose distribution. Figure 7(b) shows the film taken with the static MLC aperture and the moving motion platform. Here the acquired dose distribution is blurred; this is the result of convolution of the static dose distribution with the motion kernel. Figure $7(\mathrm{c})$ shows the film taken with the tracking MLC aperture and the moving motion platform. It can be seen qualitatively that the dose distribution shown on this film is comparable to the desired dose distribution in figure 7(a) and that MLC tracking has removed the areas of under-dose and over-dose caused by the motion. It can also be seen that the edges of the MLC leaves in this tracking film are not as well defined as those in the reference static film; this is because of the finite leaf width which leads to this effect when the MLC leaves are re-fitted to the adjusted contour to account for motion perpendicular to the direction of leaf travel. The blurring at the edges can also be attributed to the linear motion of the MLCs and the non-linear motion of the motion platform between the MLC position updates which were performed every $1 \mathrm{~s}$.

Figure 8 shows percentage difference plots from the data for patient 3. Figure 8(a) shows the percentage difference between the four-bank no-tracking film and the fourbank reference film. Here large areas of under- and over-dose are observed. Figure 8(b) shows the percentage difference between the four-bank tracking film and the four-bank reference film and the it can be seen qualitatively that MLC tracking has substantially 


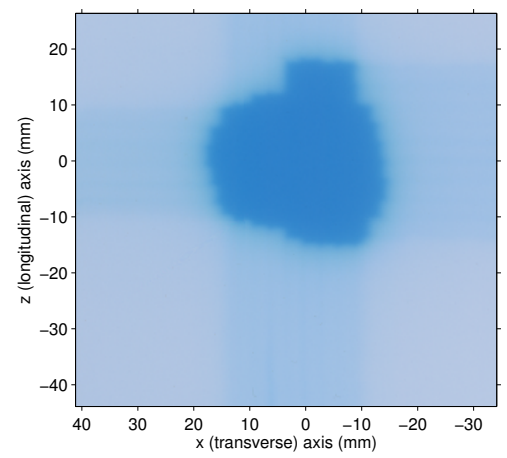

(a)

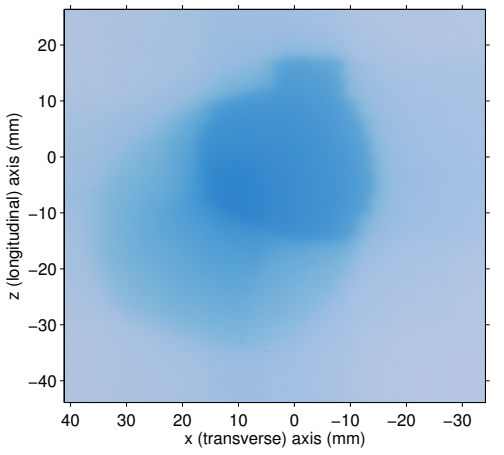

(b)

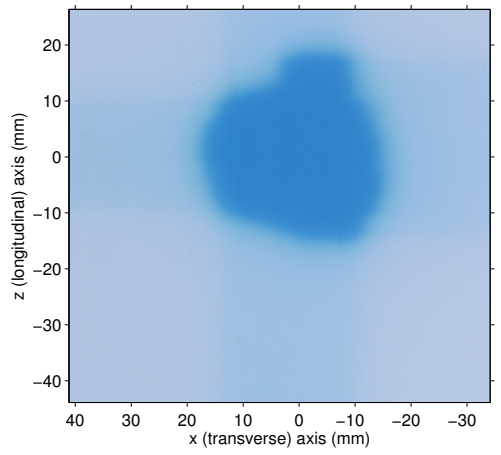

(c)

Figure 7. Gafchromic films of the four-bank aperture for patient 3. Figure 7(a) is the reference film measurement, figure $7(\mathrm{~b})$ is the no-tracking film measurement and figure $7(\mathrm{c})$ is the MLC-tracking film measurement.

improved the percentage difference from the reference; here the areas of under- and overdose are much smaller. Figure $8(\mathrm{c})$ is analogous to Figure $8(\mathrm{~b})$ but for the two-bank tracking and qualitatively the results appear very similar to the results for four-bank tracking. The results for the root-mean-square percentage difference are given in table 1, for both four-bank and two-bank tracking, for all apertures measured. Calculation of percentage difference in areas of steep dose gradient is not standard clinical practice, therefore the widely-used gamma test was performed, and the results of this are also given in table 1. It can be seen that for all apertures, both two-bank and four-bank, a reduction in the RMS percentage difference was seen with MLC tracking compared with no tracking, and very little noticeable difference was observed when four-banks rather than two-banks were used. The results of the gamma analysis show that the dosimetric differences between two-bank and four-bank tracking are clinically irrelevant: with the exception of the two-bank square aperture, all MLC-tracking films had $100 \%$ pass rate compared with an average pass rate of $68.8 \%$ without tracking.

Table 1. Summary of results from film analysis.

\begin{tabular}{llllll}
\hline \multirow{2}{*}{ Aperture } & Number of & \multicolumn{2}{c}{ RMS difference (\%) } & \multicolumn{2}{c}{$\gamma$-test $(3 \% / 3 \mathrm{~mm}$ ) pass rate $(\%)$} \\
& MLC banks & No tracking & MLC Tracking & No tracking & MLC Tracking \\
\hline \multirow{2}{*}{ Square } & Two & 7.9 & 2.6 & 70.1 & 99.7 \\
& Four & 9.8 & 1.9 & 68.4 & 100.0 \\
Patient 1 & Two & 9.1 & 2.0 & 64.4 & 100.0 \\
& Four & 9.7 & 1.5 & 64.7 & 100.0 \\
Patient 2 & Two & 8.6 & 2.1 & 69.6 & 100.0 \\
& Four & 10.1 & 2.1 & 66.9 & 100.0 \\
Patient 3 & Two & 7.3 & 1.4 & 74.3 & 100.0 \\
& Four & 9.4 & 1.8 & 72.3 & 100.0 \\
\hline
\end{tabular}




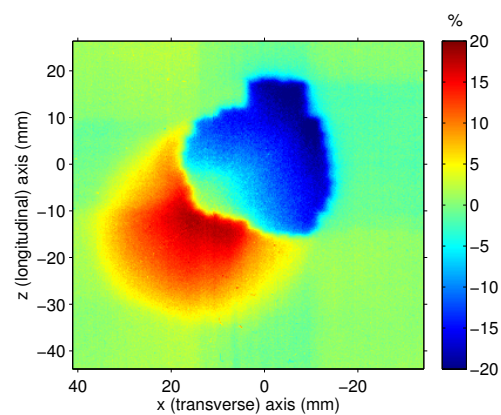

(a)

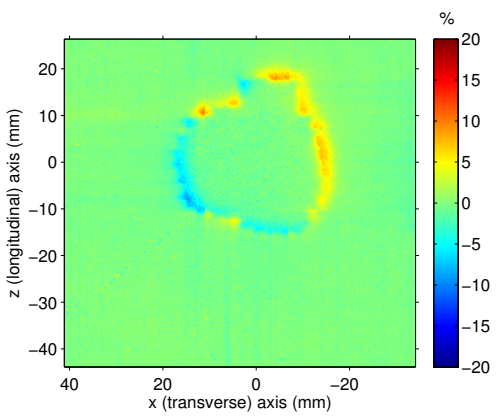

(b)

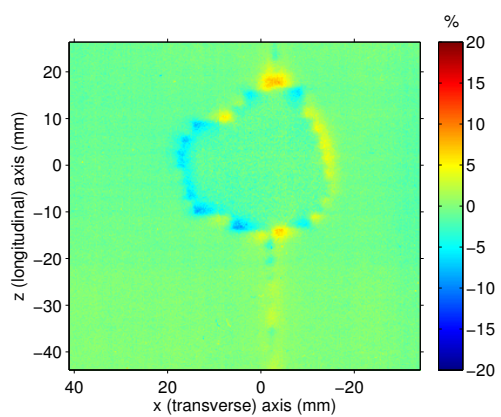

(c)

Figure 8. Percentage difference plots for patient 3. Figure $8(a)$ is the percentage difference between the four-bank reference film and the four-bank no-tracking film. Figure $8(\mathrm{~b})$ is the percentage difference between the four-bank MLC-tracking film and the four-bank reference film, and Figure $8(\mathrm{c})$ is the percentage difference between the two-bank MLC-tracking film and the two-bank reference film.

\subsection{Comparison of the $M L C$ velocity required for two-bank and four-bank $M L C$ tracking}

Figure 9(a) shows the absolute value of the maximum leaf velocity found within any of the 96 leaves for each sample of the 2D breathing cycle considered in these MLC tracking experiments. It can be seen that for all apertures, the use of four-banks for tracking rather than the conventional two-banks has led to a substantial decrease in the leaf velocity required over the breathing cycle, with the largest difference in leaf velocity seen for the square aperture. Figure 9(b) shows the maximum leaf velocity required as a function of the maximum distance found between adjacent leaves in the treatment aperture. As is expected, the leaf velocity required increases with increased maximum distance between adjacent leaves $\left(\mathrm{R}^{2}=0.93\right)$. These results are for a time period of $20 \mathrm{~s}$, and if a more realistic time period of $4 \mathrm{~s}$ was used, and the updates performed at $5 \mathrm{~Hz}$, i.e. $0.2 \mathrm{~s}$ intervals, the same graph shown in figure 9 (a) would be observed except with different scales on the axes. The x-axis of the graph would now be in the range 0 to $4 \mathrm{~s}$ and the $\mathrm{y}$-axis would be multiplied by a factor of 5 , i.e. with the current maximum leaf velocity of $20 \mathrm{~mm} \mathrm{~s}^{-1}$, the MLC tracking delivery would not be possible for the apertures studied without beam-holds. The amplitudes of motion used in this study do represent the extremes of motion observed in lung cancer patients, and can be considered a worst-case scenario.

\section{Discussion}

Experimental work has been performed that has answered questions regarding the benefit of an additional set of MLC banks and the advantages for MLC tracking of respiratory motion. The equipment that was used to answer these questions was shown to be unsuitable for clinical use in its current form, with a relatively large latency time 


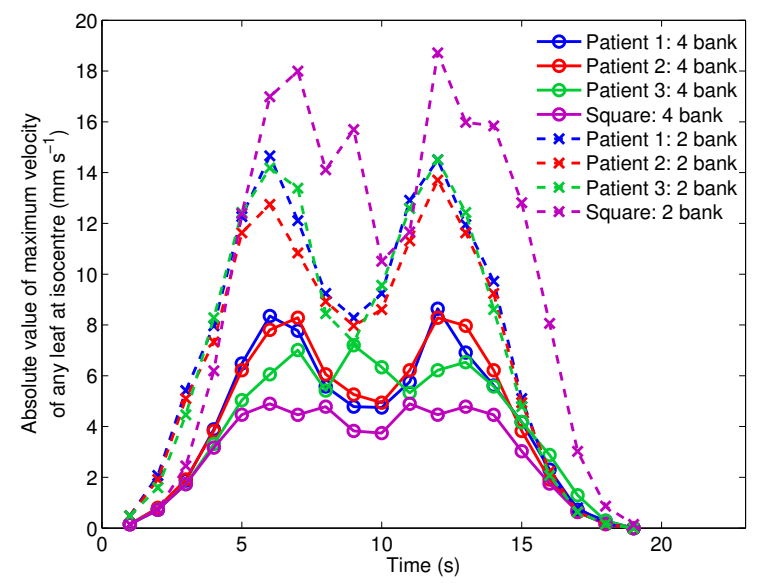

(a)

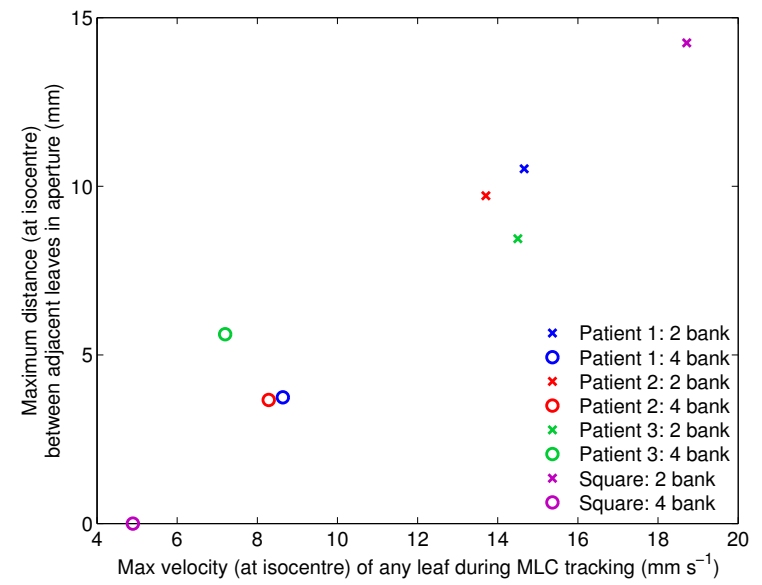

(b)

Figure 9. Results from the comparison of the MLC leaf velocity required for fourbank and two-bank tracking. Figure 9(a) shows the maximum absolute MLC leaf velocity of any of the 96 leaves during the breathing cycle for the motion studied, and figure $9(\mathrm{~b})$ shows the maximum leaf velocity required as a function of the maximum distance found between two adjacent leaves within the treatment aperture.

and low frequency at which MLC positions could be updated. The latency time of $1.0 \mathrm{~s}$ for this prototype system is greater than those reported for other MLC tracking systems including $0.62 \mathrm{~s}$ (Krauss et al. 2012) for a Siemens dMLC tracking system and $0.14 \mathrm{~s}$ for a Varian dMLC tracking system (Ravkilde et al. 2011). This relatively large latency would be prohibitive for the use of predict-ahead algorithms, where the prediction error has been shown to increase with increased look-ahead time by both Krauss (2011a) and Ruan (2010). However, as a sinusoidal motion was investigated in this study, it was possible to completely remove this $1.0 \mathrm{~s}$ latency and study the dosimetric differences between two-bank-MLC and four-bank-MLC tracking. The low frequency at which MLC positions could be updated meant that it was only possible to investigate respiratory-motion trajectories with artificially long time periods. Despite this, the study has indicated that four-bank-MLC tracking will be more efficient than two-bank-MLC tracking regardless of the time period of motion. To perform MLC tracking on clinically relevant motion trajectories, the current update frequency of 1 $\mathrm{Hz}$ should ideally be increased to at least $5 \mathrm{~Hz}$, and for the Siemens real-time dMLC tracking system, Tacke (2010) reports an update frequency of $10 \mathrm{~Hz}$. Further work will include optimising the tracking control system software to decrease the latency time and increase the update frequency, to explore MLC tracking with a four-bank-MLC for realistic, irregular patient motion.

For the particular respiratory motion investigated in this study, of equal amplitude in two orthogonal directions, the use of four-banks rather than two was shown to substantially decrease the maximum leaf velocity required for all apertures considered. This reduction in leaf velocity was greatest for the square aperture, for which when four- 
banks are used for tracking, each pair of banks can be used to track each orthogonal direction. Even with the unrealistic time period chosen in this study, the velocities required for two-bank tracking were approaching the maximum possible velocity of the MLC. If a more clinically relevant time period had been chosen for tracking, multiple radiation beam pauses would have been required for the leaves to reach their positions, which is one of the problems for two-bank MLC tracking as reported by Yoon (2011). In their study, they suggest the alternative of using a moving average algorithm for tracking the motion perpendicular to the direction of leaf travel, which they showed increased delivery efficiency whilst maintaining sufficient dosimetric accuracy. Another approach for increasing delivery efficiency for tracking techniques has been the development of the Vero system (Nakamura et al. 2010), which is equipped with a gimballed linacMLC assembly, which can pan and tilt $44 \mathrm{~mm}$ away from the beam axis, offering the advantage of decoupling the motion of the MLCs for forming the treatment apertures and modulating the fluence, from the bulk motion of the gimballed head which can be adjusted to track the moving target (Depuydt et al. 2011). This is a potential competitor to the four-bank MLC for delivery efficiency, with the disadvantage being that it must be purchased as a stand-alone system and cannot be retrofitted to an existing linear accelerator.

The experiments comparing dosimetric accuracy of two-bank-MLC and four-bankMLC tracking showed very little difference between the two techniques, with both techniques able to reconstruct the reference dose distribution to a clinically acceptable level. This result could be attributed to the very fine leaf width that the AccuLeaf mMLC device benefits from; it is possible that if a more typical MLC leaf width were used, for example, 5-10 mm, the differences between two-bank and four-bank tracking would be more pronounced, but it was not possible to address this question in this study, as a four-bank-MLC with this leaf width does not exist. As with the results for MLC leaf velocity required, the square aperture showed the largest benefit from the use of four-banks rather than two, as with this aperture design, for any shift in the beam's eye view, the four-banks can always recreate the same width square, which is not possible with the two-bank square aperture. This is reflected in the results, with a percentage decrease in the RMS \% difference value for no-tracking and MLC-tracking of $81 \%$ with four-banks (9.8\% to $1.9 \%$ ) and $67 \%$ with two-banks ( $7.9 \%$ to $2.6 \%$ ), which was the greatest difference between the two-bank and four-bank apertures observed in this dosimetric comparison.

This study concentrated on conformal treatment apertures at a static gantry angle, and the four-bank apertures were designed by arranging the leaves such as to minimise the distance between adjacent MLC leaves in each bank, hence reducing the leaf velocities encountered during tracking. An increasing number of lung cancer radiotherapy treatments are now performed with intensity-modulated radiotherapy or volumetric modulated arc therapy, therefore a comparison of two-bank-MLC versus four-bank-MLC tracking would be interesting for these delivery methods. For such a study to take place, and to maximise the benefit of four-bank-MLC tracking in terms 
of delivery efficiency, it would be advantageous to include the extra degrees of freedom from the extra MLC banks into the treatment plan optimisation process, with some prior-knowledge of the dominant axis of motion on a patient-by-patient basis. This is beyond the scope of this study, but would be an interesting topic to address in further work.

\section{Conclusion}

In this study:

(i) Experiments were presented that compared for the first time conventional two-bank and novel four-bank MLC tracking of respiratory motion with realistic conformal treatment apertures.

(ii) It has been shown that the MLC leaf velocity required is substantially reduced when four-banks rather than two-banks are used for tracking a 2D respiratory motion, indicating that the use of four-banks can increase treatment efficiency for dMLC tracking techniques.

(iii) The dosimetric differences observed between four-bank and two-bank MLC tracking were minimal, with both tracking techniques able to reconstruct a dose distribution with clinically acceptable differences from the reference dose distribution.

\section{Acknowledgements}

The authors would like to thank Chris Bunton, Young Lee and Jim Warrington from the Joint Department of Physics and Azimuth Therapy Ltd for their help and advice with this project.

\section{References}

Depuydt T, Verellen D, Haas O, Gevaert T, Linthout N, Duchateau M, Tournel K, Reynders T, Leysen K, Hoogeman M, Storme G \& De Ridder M 2011 Geometric accuracy of a novel gimbals based radiation therapy tumor tracking system. Radiother Oncol 98(3), 365-372.

Keall P J, Sawant A, Cho B, Ruan D, Wu J, Poulsen P, Petersen J, Newell L J, Cattell H \& Korreman S 2011 Electromagnetic-Guided Dynamic Multileaf Collimator Tracking Enables Motion Management for Intensity-Modulated Arc Therapy. Int J Radiat Oncol Biol Phys 79(1), 312-320.

Krauss A, Fast M F, Nill S \& Oelfke U 2012 Multileaf collimator tracking integrated with a novel x-ray imaging system and external surrogate monitoring. Phys Med Biol 57(8), 2425-39.

Krauss A, Nill S \& Oelfke U 2011 $a$ The comparative performance of four respiratory motion predictors for real-time tumour tracking. Phys Med Biol 56(16), 5303-17.

Krauss A, Nill S, Tacke M \& Oelfke U $2011 b$ Electromagnetic real-time tumor position monitoring and dynamic multileaf collimator tracking using a Siemens 160 MLC: geometric and dosimetric accuracy of an integrated system. Int J Radiat Oncol Biol Phys 79(2), 579-87.

Liu Y, Shi C, Tynan P \& Papanikolaou N 2008 Dosimetric characteristics of dual-layer multileaf collimation for small-field and intensity-modulated radiation therapy applications. J Appl Clin Med Phys 9(2), 2709. 
Low D A, Harms W B, Mutic S \& Purdy J A 1998 A technique for the quantitative evaluation of dose distributions Medical Physics 25(5), 656-661.

Lujan A E, Balter J M \& Ten Haken R K 2003 A method for incorporating organ motion due to breathing into 3D dose calculations in the liver: sensitivity to variations in motion. Med Phys 30(10), 2643-2649.

McQuaid D \& Webb S 2006 IMRT delivery to a moving target by dynamic MLC tracking: delivery for targets moving in two dimensions in the beam's eye view. Phys. Med. Biol. 51(19), 4819-4839.

Nakamura M, Sawada A, Ishihara Y, Takayama K, Mizowaki T, Kaneko S, Yamashita M, Tanabe H, Kokubo M \& Hiraoka M 2010 Dosimetric characterization of a multileaf collimator for a new four-dimensional image-guided radiotherapy system with a gimbaled x-ray head, MHI-TM2000. Med Phys 37(9), 4684-91.

Ravkilde T, Keall P J, Højbjerre K, Fledelius W, Worm E \& Poulsen P R 2011 Geometric accuracy of dynamic MLC tracking with an implantable wired electromagnetic transponder. Acta Oncol $\mathbf{5 0}(6), 944-51$.

Ruan D 2010 Kernel density estimation-based real-time prediction for respiratory motion. Phys Med Biol 55(5), 1311-26.

Sawant A, Venkat R, Srivastava V, Carlson D, Povzner S, Cattell H \& Keall P 2008 Management of three-dimensional intrafraction motion through real-time DMLC tracking. Med. Phys. 35(5), 2050-2061.

Seppenwoolde Y, Shirato H, Kitamura K, Shimizu S, van Herk M, Lebesque J V \& Miyasaka K 2002 Precise and real-time measurement of 3D tumor motion in lung due to breathing and heartbeat, measured during radiotherapy. Int $J$ Radiat Oncol Biol Phys 53(4), 822-834.

Tacke M B, Nill S, Krauss A \& Oelfke U 2010 Real-time tumor tracking: Automatic compensation of target motion using the Siemens 160 MLC Med. Phys. 37(2), 753-761.

Webb S 2012 A 4-bank multileaf collimator provides a decomposition advantage for delivering intensitymodulated beams by step-and-shoot. Phys Med 28(1), 1-6.

Yoon J W, Sawant A, Suh Y, Cho B C, Suh T S \& Keall P 2011 Experimental investigation of a moving averaging algorithm for motion perpendicular to the leaf travel direction in dynamic MLC target tracking. Med Phys 38(7), 3924-31. 